\title{
Desmoid Type Fibromatosis of the Breast Masquerading as Breast Carcinoma: Value of Dynamic Magnetic Resonance Imaging and its Correlation
}

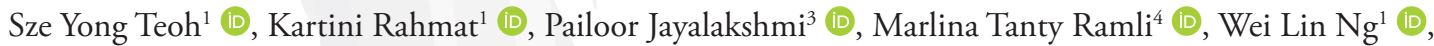 \\ Mee Hoong See ${ }^{2}$ (D), Mei Sze Teh ${ }^{2}$ (D), Anushya Vijayananthan ${ }^{1}$ (D) \\ ${ }^{1}$ Department of Biomedical Imaging, Faculty of Medicine, University of Malaya, Kuala Lumpur, Malaysia \\ ${ }^{2}$ Breast Unit Department of Surgery, Faculty of Medicine, University of Malaya, Kuala Lumpur, Malaysia \\ ${ }^{3}$ Department of Pathology, Faculty of Medicine, University of Malaya, Kuala Lumpur, Malaysia \\ ${ }^{4}$ Radiology Department, University Teknologi MARA, Selangor, Malaysia
}

\begin{abstract}
Desmoid type fibromatosis of the breasts is a rare stromal tumour of the breast that constitutes less than $0.2 \%$ of all breast tumours. Bilateral and multi-centric lesions are extremely rare, with only less than 10 cases reported in literatures. Although benign, it is locally aggressive with recurrence in up to almost one-third of the cases. This case of bilateral multi-centric breast fibromatosis was the first case in our centre. 19-year old woman, with a paternal aunt diagnosed with breast cancer at 30 -year-old, presented to our institution with the chief complaint of retracted nipples for 1 year, she denied any history of trauma to her chest. Sonography showed bilateral suspicious hypoechoic masses. MRI was performed for further evaluation due to its extensive involvement of both breasts. The aim of this review is to illustrate the main clinical, radiological and histopathological characteristics of this rare disease to increase awareness of this entity and discuss the role of MRI.
\end{abstract}

Keywords: Breast tumor, desmoid type fibromatosis, fibromatosis, magnetic resonance imaging, ultrasound

Cite this article as: Vijayananthan A, Ng WL, Teoh SY, See MH, Rahmat K, Jayalakshmi P, et al. Desmoid Type Fibromatosis of the Breast Masquerading as Breast Carcinoma: Value of Dynamic Magnetic Resonance Imaging and its Correlation. Eur J Breast Health DOI: 10.5152/ejbh.2020.5482.

\section{Introduction}

Desmoid type fibromatosis of the breast is a rare stromal tumour that constitutes less than $0.2 \%$ of all breast tumours. Although benign, this tumour is locally infiltrative with recurrence noted in up to almost one-third of the cases (1). Bilateral and multicentric lesions are extremely rare, found in only $4 \%$ of patients, with only less than 10 cases reported in literatures $(1,2)$. The imaging appearances of aggressive fibromatosis can frequently mimic invasive breast carcinoma resulting in exhaustive clinical and surgical work up.

\section{Case Presentation}

A 19-year old woman, with family history of breast cancer (paternal aunt diagnosed at 30-year-old), presented with bilateral breast lumps which were increasing in size associated with nipple retraction for 1-year. Physical examination revealed bilateral retracted nipples. There was a $4 \mathrm{~cm}$ X $5 \mathrm{~cm}$ firm, ill-defined, mobile retroareolar mass in her right breast and a $3 \mathrm{~cm} \mathrm{X} 3 \mathrm{~cm}$ firm, ill-defined, mobile in the upper outer quadrant of her left breast. There are no skin changes and no fixity to chest wall or skin. The axillary, supraclavicular and infraclavicular lymph nodes were not palpable. Breast ultrasound showed bilateral ill-defined hypoechoic masses (two on the right, one on the left) with irregular margins and posterior shadowing (Figure 1).

Initial core biopsy showed benign breast tissue. Due to imaging-histological discordance, MRI breast was performed for further evaluation. Contrast enhanced MRI of both breasts performed on a MRI 3.0 T machine (Signa ${ }^{\oplus}$ HDx GE Healthcare, Wisconsin, USA) demonstrated multiple irregular spiculated heterogeneously enhancing masses in the right breast extending from nipple to the lower outer quadrant and upper half of the breast collectively measuring $2.3 \times 5.1 \times 4.8 \mathrm{~cm}$ (Figure 2a, b). These lesions show corresponding hyperintense signals on T2/STIR and hypointense signals on T1. The nipple areolar complex was involved. There was no chest wall infiltration. Similar irregular spiculated enhancing mass is seen in the upper half of the left breast measuring approximately $2.0 \times 3.3 \times 4.1 \mathrm{~cm}$ (Figure 2c, d). Similarly, it extends into the nipple-areolar complex. Bilateral breast lesions demonstrated progressive enhancement that plateau on delayed phases consistent with Type 2 curve. 
Ultrasound guided hookwire excision biopsy were performed for three lesions (right 12:00, left 12:00 and left 6:00) and result showed desmoid type fibromatosis.

A multidisciplinary team meeting convened due to the rarity of this disease entity and the patient agreed for immediate skin sparing mastectomy and reconstruction due the local invasiveness and high recurrences of this disease despite having no metastatic potential. Patient refused autologous or implant reconstruction and opted for staged lipofilling. The final histopathology showed proliferation of stellate to spindle shape cells. Nuclear positivity for beta-catenin. Ki-67 was $<1 \%$ (Figure 3).

\section{Discussion and Conclusion}

Desmoid type fibromatosis are rare, benign and slow-growing fibroblastic neoplasm, common in women typically between the ages of 22 and 49 (1). It is characterized by being locally aggressive with fre-

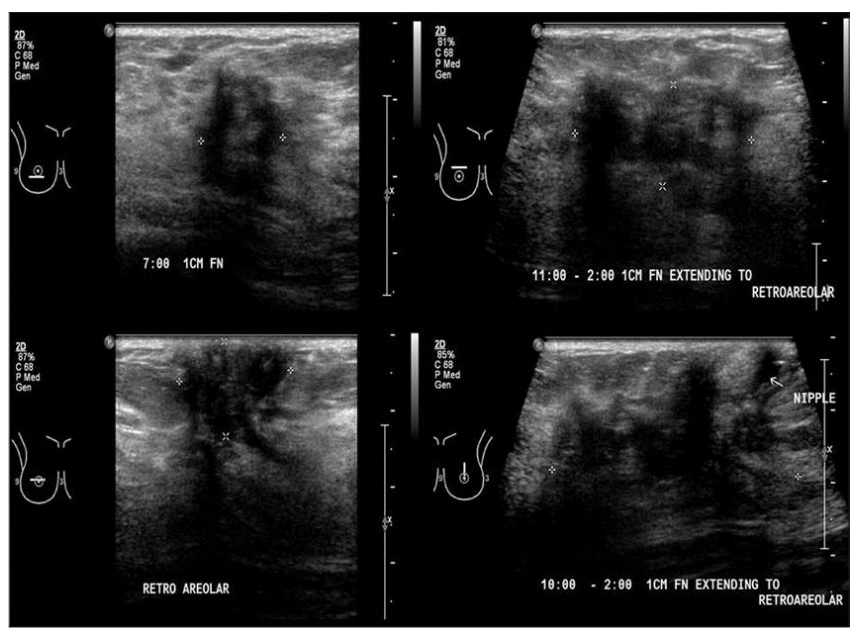

Figure 1. Irregular hypoechoic masses with posterior shadowing in the right 7 o'clock position, right upper region and retroareolar and left upper region. These bilateral breast suspicious heterogenous lesions were categorized as BIRADS 4c

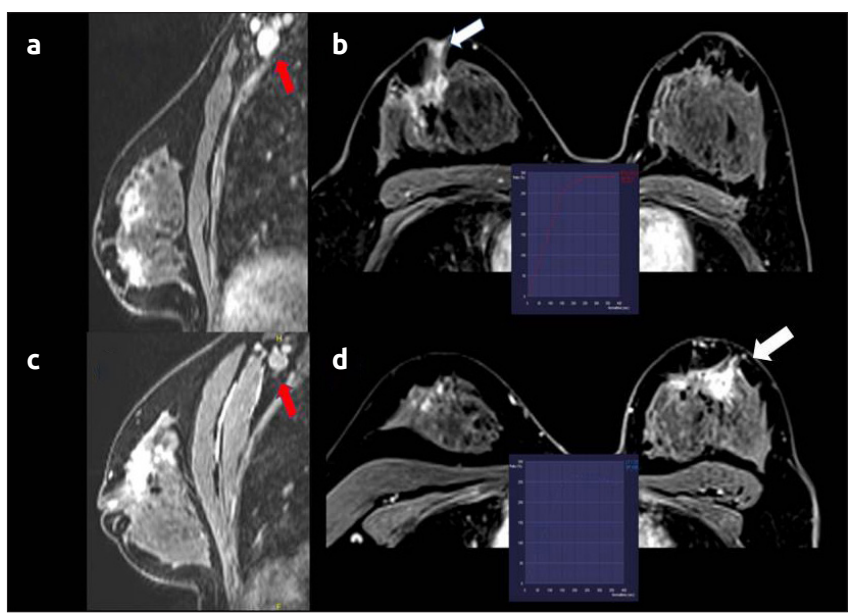

Figure 2. a-d. Dynamic post-contrast phase-3 MRI images in sagittal and axial view of the right $(a, b)$ and left $(c, d)$ breasts demonstrating heterogeneously enhancing spiculated lesions in both breasts. These lesions demonstrated type 2 kinetic curve pattern (inset). Multicentric disease were seen on MRI with involvement of the nipple-areolar complex (white arrow). Abnormal lymph nodes are also seen in both axilla (red arrow) quent local recurrence without distant metastasis. Associations with Gardner's syndrome, familial adenomatous polyposis (FAP), surgical trauma or silicone breast implants have been reported. When associated with silicone implant, fibromatosis is thought to originate from the fibrous capsule of the implant $(1,2)$.

Clinically, fibromatosis of the breast are often mobile, non-tender firm masses which can sometimes appear adherent to the chest wall. Skin dimpling and nipple retraction have been observed and these findings were similar to those found in our patient (3).

The reported cases of fibromatosis have been visible mammographically in only one third of cases. They are often irregularly shaped, non-calcified, high-density masses with spiculated margins that mimic breast cancer (1). However, mammography was not performed in our patient due to her young age and expected dense breasts. Sonographically, fibromatosis are solid, spiculated or microlobulated, hypoechoic masses with a thick echogenic rim and posterior attenuation. However, it can also present with benign features. Tethering of Cooper ligaments and involvement of the pectoralis muscle have been observed, indicating the locally aggressive nature of fibromatosis and accounting for the skin dimpling and nipple retraction $(1,2)$.

Although there is extensive literature on MRI of musculoskeletal desmoid tumours, breast imaging features on MRI have scarcely been reported. Desmoid type fibromatosis appear as ill defined, hypo- to isointense masses on T1-weighted images and as heterogeneously hyperintense masses on T2-weighted images. This findings are similar to previous studies (4-7). On dynamic MRI, they often show a gradual enhancement thought to reflect the significant amount of collagenous tissue and myxoid change of the tumor (5). The enhancement pattern can vary. There are cases of fibromatosis of the breast reported to have rapid enhancement and washout on dynamic MRI (type 2 curve), mimicking that of an invasive carcinoma. In such cases, the

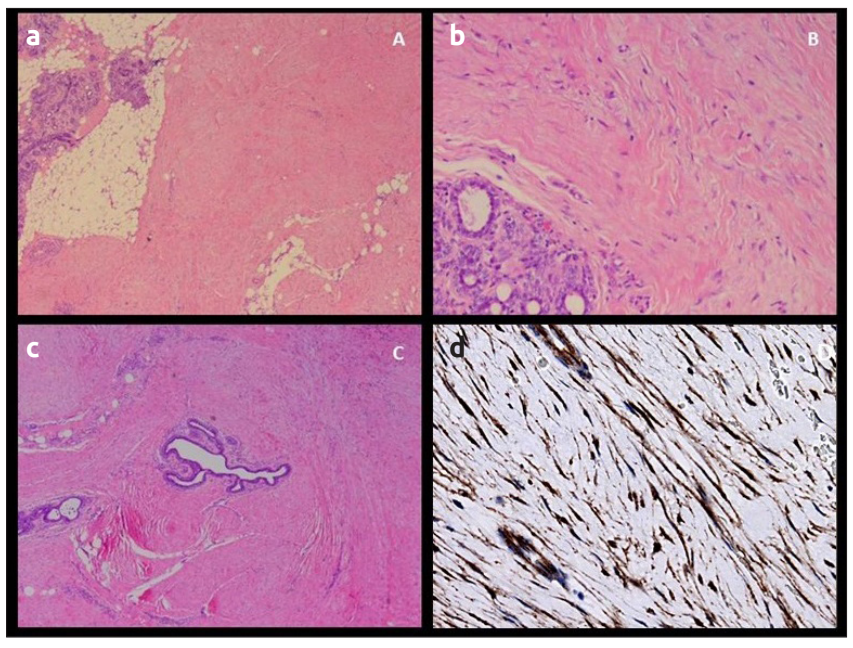

Figure 3. a-d. (a) Section of the breast showing breast tissue replaced by fibrous tissue. Proliferation of stellate to spindle shape cells in short intersecting fascicle. (H\&E stain) Magnification $x$ 40. (b) The cells have elongated, spindle shape to plump vesicular nuclei with occasional small nucleoli, pale acidophilic cytoplasm and devoid of distinct cell border. (H\&E stain) Magnification x 40. (c) Entrapped benign breast ducts and acini, mild lymphocytic infiltration and erythrocytes are seen surrounded by fibrous tissue (H\&E stain) Magnification x100. (d) Immunohistochemistry: Nuclear positivity for beta-catenin. Magnification x400.

Haematoxylin \& eosin stain (H\&E stain) 
lack of peripheral ring enhancement typical for breast carcinomas can be a differentiating feature (8). MRI also provides an accurate evaluation of pectoralis major muscle involvement which is important for surgical planning $(5,8)$.

Grossly, the appearance of desmoid type fibromatosis can vary from a well-circumscribed nodular lesion to being irregular infiltrative lesions. Histologically, the hallmark of a desmoid type fibromatosis is the presence of non-encapsulated bland-looking spindle cells organized into long sweeping and intersecting fascicles with fingerlike extensions noted at the periphery of the lesion into adjacent breast parenchyma and adipose tissue (9). In desmoid type fibromatosis, overall cellularity is low to moderate with no cytologic pleomorphism or increase in mitotic activity which are important to distinguish from metaplastic fibrosarcoma. By immunohistochemistry, the positive presence of actin and vimentin is useful for the diagnosis of desmoid type fibromatosis. Desmin is rarely positive, whereas S100 and CD34 are usually negative. $\beta$-catenin nuclear staining is also an option for diagnosis but may be only focally positive. Cytokeratin staining meanwhile is helpful in ruling out a carcinoma $(1,9)$.

Due to the local aggressiveness and high rate of recurrence, the standard treatment of desmoid type fibromatosis involves a wide surgical resection with clear margins (10). Povoski et al. (11) reported repeated excision was performed for a patient due to positive margin from primary surgery. Mastectomy was performed for our patient due to the multicentric and nipple areolar complex involvement of the disease. Clear margins were obtained.

Desmoid type fibromatosis of the breast are an unusual but distinct entity. Due to patient's young age and family's history, MRI breast is preferred over mammogram due to denser breast. It is important for all clinicians to be aware about this disease entity. Any discordance in triple assessment (clinical, radiological and pathological) for any breast symptoms must be further discussed and investigated. Although desmoid tumours have no metastatic potential, the local invasiveness of this disease may be disastrous if treatment is delayed.

Informed Consent: Written informed consent was obtained from patient who participated in this case.

Peer-review: Externally peer-reviewed.
Author Contributions: Concept - A.V.; Supervision - K.R., A.V., M.H.S., W.L.N.; Analysis and/or Interpretation - S.Y.T.; Literature Search - M.T.R.; Writing Manuscript - S.Y.T.; Critical Review - A.V., W.L.N.

Conflict of Interest: The authors have no conflicts of interest to declare.

Financial Disclosure: The authors declared that this study has received no financial support.

\section{References}

1. Glazebrook KN, Reynolds CA. Mammary fibromatosis. AJR Am J Roentgenol 2009; 193: 856-860. (PMID: 19696302) [CrossRef]

2. Wongmaneerung P, Somwangprasert A, Watcharachan K, Ditsatham C. Bilateral desmoid tumor of the breast: case seriesand literature review. Int Med Case Rep J 2016; 9: 247-251. (PMID: 27578999) [CrossRef]

3. Erguvan-Dogan B, Dempsey PJ, Ayyar G, Gilcrease MZ. Primary desmoid tumor (extraabdominal fibromatosis) of the breast. AJR Am J Roentgenol 2005; 185: 488-489. (PMID: 16037525) [CrossRef]

4. Steadman L, Crook S. Fibromatosis arising from the pectoralis major muscle mimicking breast cancer. Radiol Case Rep 2018; 13: 1174-1178. (PMID: 30233754) [CrossRef]

5. Linda A, Londero V, Bazzocchi M, Zuiani C. Desmoid tumor of the breast: radiologic appearance with a focus on its magnetic resonance features. Breast J 2008; 14: 106-107. (PMID: 18186874) [CrossRef]

6. Grimaldi MC, Trentin C, Gullo RL, Cassano E. Fibromatosis of the breast mimicking cancer: A case report. Radiol Case Rep 2018; 13: 1-5. (PMID: 29487630) [CrossRef]

7. Nakazono T, Satoh T, Hamamoto T, Kudo S. Dynamic MRI of fibromatosis of the breast. AJR Am J Roentgenol 2003; 181: 1718-1719. (PMID: 14627606) [CrossRef]

8. Mesurolle B, Leconte I, Fellah L, Feger C, Nakazono T, Kudo S. Dynamic breast MRI in recurrent fibromatosis. AJR Am J Roentgenol 2005; 184: 696-697. (PMID: 15671404) [CrossRef]

9. Aitken SJ, Presneau N, Kalimuthu S, Dileo P, Berisha F, Tirabosco R, Amary MF, Flanagan AM. Next-generation sequencing is highly sensitive for the detection of beta-catenin mutations in desmoid-type fibromatoses. Virchows Arch 2015; 467: 203-210. (PMID: 25838078) [CrossRef]

10. Benej R, Mečiarová I, Pohlodek K. Desmoid-type fibromatosis of the breast: A report of 2 cases. Oncol Lett 2017; 14: 1433-1438. (PMID: 28789360) [CrossRef]

11. Povoski SP, Marsh WL, Spigos DG, Abbas AE, Buchele BA. Management of a patient with multiple recurrences of fibromatosis (desmoid tumor) of the breast involving the chest wall musculature. World J Surg Oncol. 2006; 4: 32. (PMID: 16768799) [CrossRef] 